\title{
Impacto da fisioterapia no tratamento da vertigem
}

\author{
Impact of Physical Therapy in vertigo treatment
}

\section{Lázaro Juliano Teixeira',Gilmar Fernandes do Prado²}

\section{RESUMO}

Introdução. Vertigem é uma queixa comum na população, mas infreqüente em serviços de fisioterapia geral, talvez pelo desconhecimento de que recursos fisioterapêuticos podem ser utilizados para o tratamento das disfunções de origem otoneurológica. Objetivo. Analisar os achados clínicos e resultados de dois anos de um serviço de fisioterapia geral na abordagem fisioterapêutica de disfunções originadas por vertigem. Método. Trinta e três indivíduos com vertigem inespecífica foram tratados ao longo dos anos de 2002 e 2003. Considerou-se a melhora subjetiva descrita pelo paciente e a obtenção de testes clínicos de equilíbrio estático e dinâmico normais como critérios de melhora. Foi calculada a média da duração do tratamento em dias, e a média de sessões realizadas. Resultado. Apenas três pacientes apresentaram manobra de Dix-Hallpike positiva para vertigem paroxística posicional benigna em nossa amostra. Os demais tinham vertigem de origem cervical. Foram necessárias em média 12,12 sessões $(\mathrm{DP}=6,44)$ de fisioterapia ao longo de 24,54 dias $(\mathrm{DP}=16,64)$, para que o paciente fosse considerado tratado segundo nossos critérios, porém 19 pacientes receberam alta até no máximo 10 dias de tratamento. Conclusão. $\mathrm{O}$ tratamento de indivíduos com queixas de vertigem por meio da Fisioterapia se mostrou como um recurso efetivo, eficiente e seguro.

Unitermos. Fisioterapia (especialidade), Otorrinolaringopatias, Reabilitação, Vertigem.

Citação. Teixeira LJ, Prado GF. Impacto da fisioterapia no tratamento da vertigem.

Trabalho realizado na Universidade Federal de São Paulo, São Paulo-SP, Brasil.

1. Fisioterapeuta, Mestre em Ciências pela Universidade Federal de São Paulo-Unifesp. Prefeitura Municipal de Balneário Camboriú, Camboriú-SC, Brasil.

2. Neurologista, Doutor, Professor Adjunto da Unifesp, São Paulo-SP, Brasil.

\section{SUMMARY}

Introduction. Vertigo is a common complaint in the population, but infrequent in physiotherapy services in general, perhaps by the lack of knowledge that physiotherapeutic resources can be used for the origin otoneurological disorders treatment. Objective. To analyze the clinical findings and therapeutic results of two-year experience of a general physiotherapy department treating disorders caused by vertigo. Method. Thirty-three individuals with nonspecific vertigo were treated over the years of 2002 and 2003. Subjective improvement reported by the patient and normal results for clinical test of static and dynamic balance were considered as improvement criteria. The average duration of the treatment in days, as well as the average number of sessions were calculated. Results. Only three patients were Dix-Hallpike maneuver were positive for benign positional paroxysmal vertigo in our sample. The others had cervicogenic vertigo. An average of 12.12 sessions $(\mathrm{SD}=6.44)$ of physiotherapy were required throughout 24.54 days $(\mathrm{SD}=16.64)$ for the patient to be considered treated according to our criteria; however, 19 patients were discharged within a period no longer than 10 days of treatment. Conclusions. The treatment of individuals with vertigo complaints by means of physiotherapy was considered as an effective, efficient and safe resource.

Keywords. Physical Therapy Modalities, Otorhinolaryngologic Diseases, Rehabilitation, Vertigo.

Citation. Teixeira LJ, Prado GF. Impact of Physical Therapy in vertigo treatment.
Endereço para correspondência:

R. Ana G. Pereira, 167 CEP 88340-000, Camboriú-SC, Brasil. E-mail: lazarojt@terra.com.br

Artigo Original Recebido em: 04/11/2008 Revisado em: 05/11/2008 a 12/04/2009

Aceito em: 13/04/2009

Conflito de interesses: não 


\section{INTRODUÇÃO}

Vertigem é uma sensação rotatória do paciente em relação ao meio ambiente ou vice-versa, normalmente acompanhada de náuseas, vômitos e outros sinais e/ou sintomas neurovegetativos. Além do sintoma vertigem (sensação rotatória), alguns pacientes experimentam a sensação de movimento (oscilação, balanço, flutuação), termo designado como "tontura". Já o termo "tontura rotatória" tem o mesmo significado de vertigem ${ }^{1}$.

As tonturas ,incluindo vertigem, são queixas muito comuns, afetando aproximadamente $20-30 \%$ da população ${ }^{2}$. A prevalência de queixas de desequilíbrio na população acima dos 65 anos chega a $85 \%{ }^{3}$, estando associada a várias etiologias e pode se manifestar como desequilíbrio propriamente dito, desvio de marcha, instabilidade, náuseas e quedas freqüentes, que nesta faixa etária pode trazer graves conseqüências, como a fratura de colo de fêmur, pois também é muito mais prevalente a osteoporo$\mathrm{se}^{4}$.

Embora freqüente, a vertigem como queixa primária não é comum na clínica geral do fisioterapeuta, exceto em serviços multidisciplinares especializados ou consultórios com enfoque voltado para distúrbios funcionais relacionados à otoneurologia. A tontura pode afetar profundamente a qualidade de vida do indivíduo acometido, havendo vários instrumentos de avaliação e medida do distúrbio e do comprometimento na vida diária disponíveis aos profissionais da saúde $\mathrm{e}^{5,6}$.

As vertigens podem ser classificadas conforme a topografia da lesão em: periférica sensorial (lesão no aparelho vestibular como a doença de Ménière ou por drogas ototóxicas); periférica neural (nos nervos vestibulares, como neurite vestibular); central (no sistema nervoso central como tumores, epilepsia ou esclerose múltipla); mista (lesões periféricas e centrais concomitantes como insuficiência vértebrobasilar ou doenças metabólicas); ou indeterminada, como a psicogênica e cervicogênical.

Uma coleta detalhada da história da doença é importante, e dados como o tempo de surgimento dos sintomas, duração das crises de vertigem, tipo de vertigem e relação com perda auditiva durante as crises podem ser parâmetros que auxiliarão no diagnóstico clínico, direcionando para o tratamento mais adequado ${ }^{7}$. O impacto da vertigem na vida do doente pode ser mensurado através do Inventário das Deficiências da Vertigem validado para a população brasileira ${ }^{8}$.
Várias propostas de intervenção fisioterapêutica estão disponíveis na literatura desde que o médico Cawthorne ${ }^{9} \mathrm{e}$ o fisioterapeuta Cooksey ${ }^{10}$ propuseram um protocolo para o tratamento de indivíduos com vertigem. Mais tarde, Norré et al. propuseram exercícios de habituação vestibular, que consistem basicamente na repetição do movimento provocativo da vertigem ${ }^{11}$. Propostas menos acessíveis utilizam até mesmo recursos de realidade virtual para treino do equilíbrio através de mecanismos de habituação e compensação ${ }^{12}$.

Os objetivos da fisioterapia para o tratamento das vertigens ou tonturas são: treinar a coordenação dos movimentos oculares, o equilíbrio estático e dinâmico, melhorar o condicionamento físico geral $\mathrm{e}$ possibilitar ótimo desempenho nas atividades funcionais ${ }^{13}$.

A vertigem posicional paroxística benigna (VPPB) é o tipo de vertigem posicional mais freqüente $^{14,15}$. O diagnóstico é clínico e o tratamento é feito através de movimentos específicos com a cabeça, chamados de manobras de reposicionamento, entre elas a Manobra de Epley ${ }^{16,17}$.

Para outras vertigens do tipo sensorial, e ainda de origem neural, métodos de cinesioterapia foram avaliados por Hillier e Hollohan, que demonstraram eficácia do procedimento em relação a grupos controles em uma recente revisão sistemática de estudos clínicos controlados ${ }^{18}$.

Porém o tipo de vertigem mais comum em ambulatórios gerais de fisioterapia provavelmente seja a indeterminada, também chamado de vertigem cervicogênica. Os estudos clínicos sobre vertigem cervical são prejudicados pela inabilidade de se confirmar o diagnóstico, pela falta de testes laboratoriais que a comprovem e pela inexplicável discrepância entre indivíduos com intensa dor e disfunção cervical sem vertigem e pacientes com queixas de vertigem importante com moderada dor cervical ${ }^{19}$. Mesmo assim os mesmos autores admitem que portadores deste distúrbio podem melhorar dramaticamente com fisioterapia.

Numa revisão sistemática sobre estudos clínicos com terapia manual para tratar vertigem cervical foi observada melhora significativa dos sinais e sintomas relacionados a dor cervical e vertigem ${ }^{20}$.

O presente estudo apresenta um relato de experiência de um serviço de Fisioterapia, que atende pacientes com queixa de vertigem indeterminada, sendo nosso objetivo analisar os principais recursos 
fisioterapêuticos requeridos para o tratamento de pacientes com vertigem e seus resultados terapêuticos.

\section{MÉTODO}

\section{Amostra}

Este estudo analisou retrospectivamente 33 casos, atendidos de abril de 2002 a julho de 2003 . A coleta dos dados foi feita retrospectivamente, baseada nos prontuários dos pacientes. Os indivíduos eram encaminhados em sua maioria por um médico otorrinolaringologista ou neurologista. O projeto aprovado pelo Comitê de Ética em Pesquisa da UNIFESP (0427/09).

Este estudo analisou retrospectivamente 33 casos, atendidos de abril de 2002 a julho de 2003. A coleta dos dados foi feita retrospectivamente, baseada nos prontuários dos pacientes. Os indivíduos eram encaminhados em sua maioria por um médico otorrinolaringologista ou neurologista. O projeto aprovado pelo Comitê de Ética em Pesquisa da UNIFESP $(0427 / 09)$.

\section{Procedimento}

Inicialmente foi realizada uma consulta fisioterapêutica, na qual foram coletados os dados pessoais, queixa e história da disfunção, dados do exame clínico com ênfase na amplitude de movimento da coluna cervical, testes clínicos de equilíbrio estático (Romberg) e dinâmico, através da estabilidade e independência funcional durante trocas de posturas, em diferentes posturas (sentado, em pé, de joelhos e semi-joelhos), marcha e de vertigem posicional ou teste de Dix-Hallpike, conforme a queixa, pelo fisioterapeuta responsável (LJT).

Durante a atividade de trabalho fisioterapêutico em 2002 e 2003, não havia intenção de levantamento de dados para pesquisa. $\mathrm{O}$ registro nos prontuários foi feito baseado em testes clínicos convencionais, como postura estática em pé, sentado, de joelhos e semi joelhos, Sinal de Romberg e marcha, estabilidade e independência funcional para trocas de posturas, amplitude de movimento da coluna cervical. Não houve mensuração destes parâmetros em todos os pacientes e o registro era feito no prontuário apenas no caso de existir alterações nos testes acima.

Os atendimentos, incluindo avaliação e tratamento fisioterapêutico, foram realizados no serviço de fisioterapia de Brusque-SC, Brasil pelo mesmo profissional.

O tratamento foi conduzido em diferentes etapas. Para os participantes que, na avaliação, apresentavam nistagmo posicional característico de VPPB durante a manobra de Dix-Hallpike, foi realizada a manobra de Epley ${ }^{16}$. Essa manobra consiste em movimentos orientados da cabeça no plano do canal semicircular afetado, visando deslocamento de cristais de cálcio (otocônias) presentes, na maioria das vezes, no canal semicircular posterior. Sua simplicidade, facilidade de aplicação e efetividade foram demonstradas em revisões de estudos clínicos randomizados ${ }^{21}$.

Pacientes sem nistagmo posicional e com suspeita de vertigem cervicogênica foram tratados, inicialmente, através de terapia manual, que consistia de massoterapia cervical, mobilização (técnicas de baixa velocidade e pequena ou grande amplitude), e alongamento passivo da coluna cervical. O relaxamento era induzido concomitantemente à terapia manual, visando principalmente desativação da formação reticular central e sua possível influência no quadro tensional muscular e desconfortos da vertigem com ela relacionada ${ }^{22}$.

Um programa de exercícios sensoriomotores também foi empregado de forma semelhante à descrita por Treleaven que propõe um atendimento personalizado para tratar os distúrbios do equilíbrio associados com disfunção cervical através de exercícios ativos ${ }^{23}$.

Além disso, realizavam-se exercícios baseados em protocolos de compensação e habituação vestibular desenvolvidos por Norré et al. ${ }^{24}$ que eram prescritos individualmente. No decorrer das sessões, após a readequação da função do equilíbrio corporal, foram realizados alongamentos ativos e autoassistidos da musculatura cervical e da cadeia muscular posterior, visando relaxamento geral, melhora da amplitude dos movimentos cervicais e início do treinamento sensório motor da musculatura cervical e motricidade óculo-cervical. Todos os indivíduos faziam uso de medicação conforme prescrição médica.

O resultado esperado após o tratamento fisioterapêutico era a ausência de queixas subjetivas de vertigem referidas espontaneamente e testes clínicos de equilíbrio estático e dinâmico, coordenação e propriocepção normalizados. Quando o paciente não apresentava mais queixas ou alterações do equilíbrio corporal, recebia alta do tratamento fisioterapêutico, era contra-referenciado ao médico e orientado a iniciar prática de atividade física de forma continuada. Contato telefônico posterior era mantido com alguns pacientes para confirmar a manutenção da melhora. 
Os dados dos pacientes foram coletados de seus prontuários durante o ano de 2007. Foram descritos os principais achados clínicos dos indivíduos baseando-se na Classificação Internacional de Funcionalidade (CIF), proposta pela Organização Mundial da Saúde com a intenção de padronização dos diagnósticos funcionais ${ }^{25}$. A ferramenta é dividida em duas partes, cada uma com dois componentes: Parte 1: Funcionalidade e incapacidade, a) Funções do corpo e b) Atividades e Participação; Parte 2: Fatores contextuais: c) fatores ambientais e d) Fatores pessoais. Neste estudo utilizamos apenas a primeira parte da CIF, relacionada às funções corporais, atividades e participações.

\section{Análise Estatística}

Os resultados da média, mediana e desvio padrão do número de dias de tratamento e número de sessões realizadas foram analisados descritivamente, através do programa SPSS for Windows, versão 14.0. Não foram realizados testes estatísticos, uma vez que não houve comparações.

\section{RESULTADOS}

Após os atendimentos constatou-se que indivíduos com queixas de vertigem em tratamento fisioterapêutico não possuem alterações em estruturas corporais, mas há várias alterações possíveis das funções corporais, que afetarão conseqüentemente as atividades e participações. As alterações percebidas durante o tratamento fisioterapêutico são listadas na Tabela 1, seguindo o modelo da CIF. Todos os 33 indivíduos possuíam quadro de vertigem inespecífica. Trinta deles possuíam diagnóstico médico de vertigem cervicogênica. Três pacientes apresentaram nistagmo característico após o teste de Dix-Hallpike, caracterizando a VPPB (indivíduos 3, 16 e 17). Um paciente (19) não tinha um diagnóstico definido, sendo interrompido seu tratamento sem alteração no quadro clínico e funcional (Tabela 2). Emmédiaforamrealizadas $12,12 \pm 6,44$ sessões defisioterapiaaolongode24,54 $\pm 16,64$ diasemmédia(Tabela3).

Constatou-se que dezenove indivíduos $(57,57 \%)$ fizeram 10 sessões de fisioterapia, ou seja, mais da metade dos tratamentos duraram o número de sessões padronizados pelos seguros de saúde, indicando um possível viés de amostra. Ao excluir os pacientes com VPPB do cálculo da média, obtivemos uma média de 25,66 dias de tratamento e de 12,83 sessões.

Quanto à satisfação e melhora do quadro clínico dos participantes, verificou-se subjetivamente que os pacientes se tornavam mais confiantes para realização de suas atividades de vida diária, se sentiam mais contentes e algumas vezes motivados a iniciar práticas corporais ou atividades físicas antes não imaginadas.

Tabela 1. Listagem das principais funções corporais e atividades e participações limitadas no paciente com queixa de vertigem encaminhado para a Fisioterapia.

\begin{tabular}{|c|c|c|}
\hline Código CIF & $\begin{array}{l}\text { Classificação } \\
\text { detalhada }\end{array}$ & Descrição \\
\hline b210-b249 & Funções sensoriais & \\
\hline $\mathrm{b} 2152$ & $\begin{array}{l}\text { Funções dos músculos } \\
\text { externos do olho }\end{array}$ & $\begin{array}{l}\text { Funções dos músculos que são } \\
\text { utilizados para olhar em dife- } \\
\text { rentes direções, para seguir um } \\
\text { objeto que se move no campo } \\
\text { visual, produzir movimentos } \\
\text { sacádicos que localizam um } \\
\text { objeto em movimento e para } \\
\text { fixar o olho. }\end{array}$ \\
\hline b2351-b2352 & $\begin{array}{l}\text { Função vestibular de } \\
\text { equilíbrio e do movi- } \\
\text { mento }\end{array}$ & $\begin{array}{l}\text { Funções sensoriais do ouvido } \\
\text { interno relacionadas à determi- } \\
\text { nação do equilíbrio do corpo e } \\
\text { à determinação do movimento } \\
\text { do corpo, incluindo sua dire- } \\
\text { ção e velocidade. }\end{array}$ \\
\hline b2401 & Tontura & $\begin{array}{l}\text { Sensação de movimento envol- } \\
\text { vendo a pessoa ou o próprio } \\
\text { ambiente; sensação de rodar, } \\
\text { balançar ou inclinar. }\end{array}$ \\
\hline b2403 & $\begin{array}{l}\text { Náusea associada à ton- } \\
\text { tura ou vertigem }\end{array}$ & $\begin{array}{l}\text { Sensação de desejo de vomi- } \\
\text { tar produzida pela tontura ou } \\
\text { vertigem }\end{array}$ \\
\hline b730-b749 & Função neuromuscular & \\
\hline b7350 b7355 & $\begin{array}{l}\text { Tônus de músculos } \\
\text { isolados e grupos de } \\
\text { músculos; Tônus dos } \\
\text { músculos do tronco }\end{array}$ & $\begin{array}{l}\text { Funções relacionadas à tensão } \\
\text { presente nos músculos isolados } \\
\text { e grupos de músculos em re- } \\
\text { pouso e à resistência oferecida } \\
\text { quando se tenta mover esses } \\
\text { músculos passivamente }\end{array}$ \\
\hline b770 & Marcha & $\begin{array}{l}\text { Funções relacionadas aos pa- } \\
\text { drões de movimento como } \\
\text { andar, correr ou outros movi- } \\
\text { mentos do corpo inteiro. }\end{array}$ \\
\hline $\mathrm{d} 410$ & $\begin{array}{l}\text { Atividades e participa- } \\
\text { ções }\end{array}$ & \\
\hline d4100-d4106 & $\begin{array}{l}\text { Mudar a posição básica } \\
\text { do corpo }\end{array}$ & $\begin{array}{l}\text { Deitar-se, Agachar-se, Ajoe- } \\
\text { lhar-se, Sentar-se, Levantar-se, } \\
\text { Inclinar-se, Mudar o centro de } \\
\text { gravidade do corpo. }\end{array}$ \\
\hline $\mathrm{d} 4154$ & Permanecer em pé & $\begin{array}{l}\text { Permanecer em pé durante o } \\
\text { tempo necessário, como quan- } \\
\text { do se espera numa fila. Inclui: } \\
\text { permanecer de pé em superfi- } \\
\text { cies inclinadas, escorregadias } \\
\text { ou duras. }\end{array}$ \\
\hline d640-d650 & $\begin{array}{l}\text { Realização das tarefas } \\
\text { domésticas }\end{array}$ & $\begin{array}{l}\text { Lavar e secar roupas, Limpar } \\
\text { a cozinha e utensílios, Limpar } \\
\text { a casa, Utilizar aparelhos do- } \\
\text { mésticos, Cuidar de plantas } \\
\text { internas e externas, Cuidar de } \\
\text { animais. }\end{array}$ \\
\hline d8500-d8502 & Trabalho e emprego & $\begin{array}{l}\text { Trabalho autônomo, trabalho } \\
\text { em tempo parcial ou integral }\end{array}$ \\
\hline
\end{tabular}

Fonte: CIF - Classificação Internacional de Funcionalidade (OMS 2004) ${ }^{33}$. 
Tabela 2. Sumário dos casos.

\begin{tabular}{|c|c|c|c|}
\hline Participantes & Diagnóstico & Dias & Sessões \\
\hline 1 & Vertigem cervical & 22 & 10 \\
\hline 2 & Vertigem cervical & 20 & 20 \\
\hline 3 & VPPB (??) & 35 & 10 \\
\hline 4 & Vertigem cervical & 16 & 10 \\
\hline 5 & Vertigem cervical & 28 & 20 \\
\hline 6 & Vertigem cervical & 84 & 20 \\
\hline 7 & Vertigem cervical & 20 & 10 \\
\hline 8 & Vertigem cervical & 13 & 10 \\
\hline 9 & Vertigem cervical & 12 & 7 \\
\hline 10 & Vertigem cervical & 15 & 10 \\
\hline 11 & Vertigem cervical & 40 & 10 \\
\hline 12 & Vertigem cervical & 37 & 10 \\
\hline 13 & Vertigem cervical & 22 & 10 \\
\hline 14 & Vertigem cervical & 14 & 10 \\
\hline 15 & Vertigem cervical & 21 & 10 \\
\hline 16 & VPPB & 4 & 4 \\
\hline 17 & VPPB & 1 & 1 \\
\hline 18 & Vertigem cervical & 15 & 10 \\
\hline 19 & Vertigem cervical (??) & 70 & 30 \\
\hline 20 & Vertigem cervical & 37 & 30 \\
\hline 21 & Vertigem cervical & 42 & 20 \\
\hline 22 & Vertigem cervical & 14 & 10 \\
\hline 23 & Vertigem cervical & 21 & 10 \\
\hline 24 & Vertigem cervical & 18 & 10 \\
\hline 25 & Vertigem cervical & 23 & 10 \\
\hline 26 & Vertigem cervical & 15 & 10 \\
\hline 27 & Vertigem cervical & 24 & 10 \\
\hline 28 & Vertigem cervical & 15 & 10 \\
\hline 29 & Vertigem cervical & 21 & 10 \\
\hline 30 & Vertigem cervical & 19 & 6 \\
\hline 31 & Vertigem cervical & 32 & 20 \\
\hline 32 & Vertigem cervical & 13 & 7 \\
\hline 33 & Vertigem cervical & 27 & 15 \\
\hline
\end{tabular}

\section{DISCUSSÃO}

O presente estudo relatou a experiência do atendimento de indivíduos com queixas de vertigem indeterminada em um serviço de fisioterapia geral através de técnicas de terapia manual e cinesioterapia sugerindo que o tratamento fisioterapêutico possa auxiliar na evolução de pacientes com vertigem.

Na prática, percebe-se que além do envolvimento da função vestibular, dos movimentos dos olhos, das queixas de tontura, náusea e vômito, é comum encontrar aumento de tônus da região cervical ou de toda a cadeia muscular posterior. O conjunto de sinais e sintomas predispõe a mudança nos hábitos de vida diária e pode comprometer as atividades de trabalho.

Embora a maioria dos indivíduos tratados fosse encaminhada com o diagnóstico de vertigem cervicogênica, muito debate existe na literatura sobre esta entidade nosológica ${ }^{19}$. De fato, muitos a consideram um diagnóstico de exclusão.

Recentemente, os resultados de um estudo clínico com 17 pacientes com vertigem de origem cervical, tratados com terapia manual e cinesioterapia, sem adotar os princípios da reabilitação vestibu$\operatorname{lar}^{26}$, mostrou que o tratamento durou em média 13 semanas (variando de 5 a 20) sendo feitas em média 13 sessões (5 a 23) no grupo tratado. O número de sessões daquele autor é semelhante ao nosso, porém o período de tratamento de nossos pacientes foi $43 \%$ menor.

Outro estudo descreveu retrospectivamente o tratamento de 116 pacientes com tontura através dos exercícios de Cawtorne e Cooksey indicados para casa e sessões remarcadas quinzenalmente durante no máximo 3 meses, totalizando 4 sessões ${ }^{27}$. Neste período $75,7 \%$ dos pacientes tiveram sucesso no tratamento (55,1\% com melhora total). Em nosso estudo necessitamos um período de tempo menor para indicarmos alta do tratamento, porém com um número maior de sessões.

Tabela 3. Estatística descritiva.

\begin{tabular}{|l|l|l|l|}
\hline Dias de tratamento & & Número de sessões & \\
\hline Média & 24,55 & Média & 12 \\
\hline Mediana & 21 & Mediana & 10 \\
\hline $\begin{array}{l}\text { Desvio padrão da } \\
\text { média }\end{array}$ & 2,90 & Desvio padrão da média & 1,12 \\
\hline Mínimo & 1 & Mínimo & 1 \\
\hline Máximo & 84 & Máximo & 30 \\
\hline Intervalo & 83 & Intervalo & 29 \\
\hline Desvio padrão & 16,65 & Desvio padrão & 6,44 \\
\hline Variância & 277,07 & Variância & 41,48 \\
\hline Curtose corrigida & 2,34 & Curtose corrigida & $-1,10$ \\
\hline $\begin{array}{l}\text { Desvio padrão de } \\
\text { curtose }\end{array}$ & 0,80 & Desvio padrão de curtose & 0,80 \\
\hline Assimetria & 2,05 & & 1,41 \\
\hline
\end{tabular}


A maioria dos atendimentos foi custeada através de planos de saúde, que restringe a intervenção fisioterapêutica a um número arbitrário de 10 sessões, as quais podem eventualmente ser estendidas conforme a interpretação do médico solicitante. Este fato, talvez tenha influenciado nossos resultados, uma vez que a prática clínica revela que 10 sessões não se relacionam necessariamente ao tempo suficiente para a melhora do quadro clínico do paciente, e embora referissem melhora, muitos pacientes podem simplesmente ter interrompido o tratamento após 10 intervenções.

Observamos que pacientes sem convênio de saúde atingiram a melhora esperada com um menor número de sessões e em menor tempo que a média, sugerindo que talvez o sistema de reembolso adotado na relação terapêutica possa influenciar para uma maior participação e comprometimento do paciente para sua melhora.

Quanto ao tratamento dos três pacientes com VPPB, originalmente Epley sugere que se façam manobras semanais até em torno da terceira semana, totalizando em média três sessões para o tratamento completo 16. No entanto, mais recentemente Chang e colaboradores demonstraram que indivíduos com VPPB apresentam também alterações do equilíbrio corporal diferentes da população sem o problema, e ao tratá-los não apenas através das manobras de reposicionamento, mas também com exercícios para melhorar a disfunção do equilíbrio, obtiveram diferenças significativas ao mensurar o equilíbrio estático e dinâmico com plataforma de força, a marcha e a intensidade subjetiva da vertigem e uma média de 9,9 sessões ao longo de um mês de tratamento ${ }^{28}$.

Estes últimos estudos vêm auxiliar na justificativa de que alguns indivíduos portadores de VPPB poderiam continuar com o tratamento para além de três sessões, como ocorreu com um dos indivíduos neste trabalho. Tal justificativa, no entanto, deve ser corroborada por outros trabalhos.

Outra forma de abordagem das tonturas e vertigens é através de exercícios designada reabilitação vestibular personalizada ${ }^{29}$. Em uma revisão de literatura $^{30}$ foi relatado que um programa de exercícios de reabilitação vestibular individualizado, incluindo atenção a fatores não vestibulares que possam afetar as funções de equilíbrio, associa-se a melhores desfechos clínicos após o tratamento. Considerando os dados, propostas e protocolos apresentados por diversos autores, para conduzir a cinesioterapia voltada às necessidades individuais, utilizamos uma combinação entre terapia manual, relaxamento, exercícios oculomotores, alongamentos e treino de equilíbrio e propriocepção.

Quanto à satisfação e melhora do quadro clínico dos participantes, verificou-se subjetivamente que os pacientes se tornavam mais confiantes para realização de suas atividades de vida diária, se sentiam mais satisfeitos e algumas vezes motivados a iniciar práticas corporais ou atividades físicas antes não imaginadas. Essa resolução da desordem do equilíbrio leva a uma melhora da independência do paciente e da vida social por meio da redução do risco de queda e pelo aumento da auto-estima. Além disso, promover uma vida ativa e social significa melhorar a saúde do paciente tanto física quanto emocionalmente, o que reduz comorbidades e a quantidade de drogas ingeridas.

\section{CONCLUSÃO}

Os comprometimentos das funções corporais e limitações das atividades e participações em pacientes com queixa de tontura são facilmente tratados através de recursos fisioterapêuticos comuns, como terapia manual cervical, exercícios para o treino do sistema vestibular, exercícios de propriocepção e equilíbrio, alongamentos e cuidados posturais. Este estudo demonstra que o tratamento de vertigem por meio de fisioterapia é efetivo, de fácil aplicação e com grande retorno em termos funcionais.

Sugere-se que fisioterapeutas dediquem-se ao tratamento dos indivíduos com queixas de vertigem e que apresentam distúrbios cinesiológico-funcionais a ela relacionados, possibilitando ampliar o campo de atuação profissional e as opções de intervenção a uma parcela importante da população afetada pela tontura.

Agradecimento: À Dra. Fernanda Pereira dos Santos Silva pela revisão crítica e contribuições ao texto deste manuscrito. À Dra. Maria Salete Dalcegio pela gentileza de permitir o acesso aos prontuários do Serviço de Fisioterapia.

\section{REFERÊNCIAS BIBLIOGRÁFICAS}

1.SBORL. Consenso sobre vertigem. Rev Bras Otorinolaringol 2000;66:10-38.

2.Neuhauser HK. Epidemiology of vertigo. Curr Opin Neurol 2007;20:40-6.

3.Simoceli L, Bittar RMS, Bottino MA, Bento RF. Perfil diagnóstico do idoso portador de desequilíbrio corporal: resultados preliminares. Rev Bras Otorinolaringol 2003;69:772-7. 
5.Berg K, Norman KE. Functional assessment of balance and gait. In: Studenski S (ed.). Clinics in Geriatric Medicine: Gait and Balance Disorders. Philadelphia: WB Saunders Company, 1996, p.705-24.

6.Horak FB. Clinical assessment of balance disorders. Gait Posture 1997;6:76-84.

7.Kentala E, Rauch SD. A practical assessment algorithm for diagnosis of dizziness. Otolaryngol Head Neck Surg 2003;128:54-9.

8.Castro ASO, Gazzola JM, Natour J, Ganança FF. Versão brasileira do Dizziness Handicap Inventory. Pro Fono 2007;19:97-104.

9.Cawthorne T. The physiological basis of head exercises. J Chart Soc Physiother 1944:106-7.

10.Cooksey FS. Rehabilitation in vestibular injuries. Proc R Soc Med 1945;39:273-8.

11.Norre ME, Beckers A. Vestibular habituation training. Specificity of adequate exercise. Arch Otolaryngol Head Neck Surg 1988;114:883-6.

12.Furman JMR, Hain TC, Paige GD. Central adaptation models of the vestibuloocular and optokinetic systems. Biol Cybern 1989;61:255-64.

13.Gill-Body K. Current concepts in the management of patients with vestibular dysfunction. PT Mag Phys Ther 2001;9:40-59.

14.Bronstein AM. Benign paroxysmal positional vertigo: some recent advances. Curr Opin Neurol 2003;16:1-3.

15.Resende CR, Taguchi CK, Almeida JGd, Fujita RR. Reabilitação vestibular em pacientes idosos portadores de vertigem posicional paroxística benigna. Rev Bras Otorinolaringol 2003;69:34-8.

16.Epley JM. The canalith repositining procedure: for treatment of the benign paroxysmal positional vertigo. Otolaryngol Head Neck Surg 1992;107:399-404.

17.Hilton M, Pinder D. Benign paroxysmal positional vertigo: A safe and effective treatment is available for this well defined condition. BMJ 2003;326:7391-673.

18.Hillier SL, Hollohan V. Vestibular rehabilitation for unilateral peripheral vestibular dysfunction (Cochrane review). Coch Datab Syst Rev 2007: 4 .
19.Brandt T, Bronstein AM. Cervical vertigo. J Neurol Neurosurg Psychiatr 2001;71:8-12.

20.Reid SA, Rivett DA. Manual therapy treatment of cervicogenic dizziness: a systematic review. Man Ther 2005;10:4-13.

21.Teixeira LJ, Machado JNP. Manobras para o tratamento da vertigem posicional paroxística benigna: revisão sistemática da literatura. Rev Bras Otorinolaringol 2006;72:130-9.

22.Treleaven J. Sensorimotor disturbances in neck disorders affecting postural stability, head and eye movement control. Man Ther 2008;13:211.

23.Treleaven J. Sensorimotor disturbances in neck disorders affecting postural stability, head and eye movement control - Part 2: Case studies. Man Ther 2008;13:266-75.

24.Norre ME, Beckers A. Vestibular habituation training: exercise treatment for vertigo based upon the habituation effect. Otolaryngol Head Neck Surg 1989;101:14-9.

25.OMS. CIF: Classificação Internacional de Funcionalidade. São Paulo: Edusp, 2004, 325p.

26.Malmström EM, Karlberg M, Melander A, Magnusson M, Moritz U. Cervicogenic dizziness-musculoskeletal findings before and after treatment and long-term outcome. Disabil Rehabil 2007;29:1193-205.

27.Pedalini MEB, Bittar RSM, Formigoni LG, Cruz OLM, Bento RF, Miniti A. Reabilitação vestibular como tratamento da tontura: experiência com 116 casos. Arq Int Otorrinolaringol 1999;3:74-8.

28.Chang W-C, Yang Y-R, Hsu L-C, Chern C-M, Wang R-Y. Balance improvement in patients with benign paroxysmal positional vertigo. Clin Rehabil 2008;22:338-47.

29.Herdman SJ, Whitney SL. Tratamento da Hipofunção vestibular. In: Herdman SJ (ed.). Reabilitação vestibular. Barueri: Manole, 2002, 388p. 30.Black FO, Pesznecker SC. Vestibular adaptation and rehabilitation. Curr Opin Otolaryngol Head Neck Surg 2003;11:355-60. 